\title{
Mutagenic Analysis of hPNMT Confirms the Importance of Lys57 and the Inhibitor Binding Site
}

\author{
Ki-Woong Jeong, Dong-II Kang, ${ }^{\dagger}$ Jee-Young Lee, and Yangmee Kim*
}

\author{
Department of Bioscience and Biotechnology, Bio/Molecular Informatics Center, Konkuk University, Seoul 143-701, Korea \\ *E-mail:ymkim@konkuk.ac.kr. \\ ${ }^{\dagger}$ Department of Chemistry, Konkuk University, Seoul 143-701, Korea \\ Received November 10, 2010, Accepted November 27, 2010
}

\begin{abstract}
In previous report, with the aid of receptor-oriented pharmacophore-based in silico screening, we characterized three novel hPNMT inhibitors (YPN010, YPN016, and YPN017) and proposed that the hydrogen bonding interaction between inhibitors and side chain of Lys57 is very important to inhibitory activity of hPNMT. To confirm the importance of Lys57, mutant with substitution of Lys57 with Ala was cloned and binding study was performed for a K57A mutant of hPNMT using STD-NMR and fluorescence experiments. The binding constants for three novel inhibitors with mutant hPNMT were dramatically decreased compared to those with wild-type protein. K57A mutant-induced conversion of noradrenaline to adrenaline was suppressed about $95 \%$ compared to wild-type hPNMT. Mutagenic analysis using a K57A mutant confirmed the importance of the Lys57 residue in binding of the inhibitor candidate to hPNMT as well as enzymatic activity of hPNMT, implying that these results are consistent with our binding model.
\end{abstract}

Key Words: PNMT, Adrenaline, Mutagenic analysis, STD NMR, In silico screening

\section{Introduction}

$N$-methylation is a prominent pathway for the metabolism of several endogenous hormones and neurotransmitters. This reaction occurs via transfer of a methyl group from $S$-adenosylL-methionine (SAM) to nucleophilic amino groups, leading to the production of $N$-methylated metabolites and $S$-adenosylhomocysteine (SAH). ${ }^{1}$ Adrenaline (or epinephrine) accounts for $5-10 \%$ of total catecholamines in the central nervous system (CNS). ${ }^{2}$ Adrenaline is synthesized in vivo from noradrenaline in a reaction catalyzed by phenylethanolamine $N$-methyltransferase (PNMT), a $30 \mathrm{kDa}$ enzyme that utilizes the cofactor SAM to methylate the amine of noradrenaline. ${ }^{3}$ It is conceivable that inhibitor of hPNMT can be used to regulate level of adrenaline within the CNS, thus providing considerable assistance in elucidating the role of central adrenaline., ${ }^{4,5}$

An extended series of investigations has focused on identi-

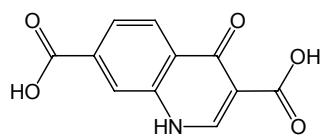

YPN010<smiles>Nc1cc(S(=O)(=O)O)c(N)c2ccc(S(=O)(=O)O)cc12</smiles>

YPN017

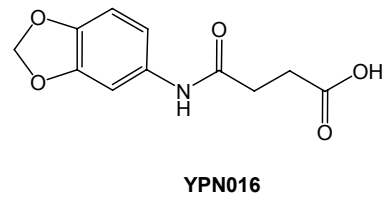<smiles>c1ccc2c(c1)CCNC2</smiles>

THIQ

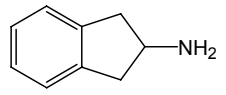

2-aminoindan
Figure 1. The chemical structures of inhibitors used in this study. fying effective substrates and inhibitors of hPNMT. However, the majority of these compounds are based on 1,2,3,4-tetrahydroisoquinoline and its analogues. ${ }^{6-10}$ We reported three potential and novel inhibitors of hPNMT (YPN010, YPN016 and YPN017) using 3D pharmacophore-based in silico screening, biophysical screening and enzymatic activity assay. ${ }^{11}$ These YPN inhibitors and known inhibitors of hPNMT such as THIQ and 2-aminoindan are shown in Figure $1 .^{8}$ These novel inhibitors comprised one hydrogen bond acceptor, one hydrogen bond donor, one lipophilic feature, and shape constraints, including a hydrogen bond between Lys57 of hPNMT and a hydrogen bond donor of the inhibitor, and stacked hydrophobic interactions between the side-chain of Phe182 and an aromatic region of the inhibitor as shown in Figure 2.

To confirm the importance of Lys 57 for interaction between hPNMT and inhibitors and verify the inhibitor binding site reported previously, mutagenic analysis and binding study using

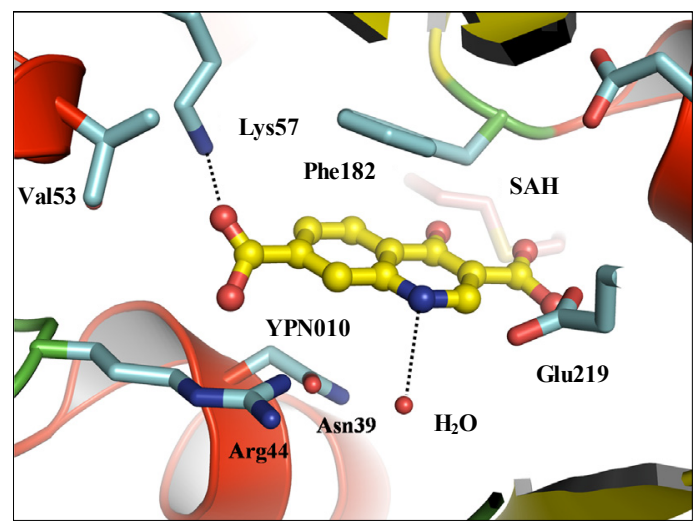

Figure 2. Interaction model of YPN010 at the active site of hPNMT. Hydrogen bonds are included in the docking model of inhibitors and hPNMT (dashed lines). 
STD-NMR and fluorescence quenching experiment were performed for a K57A mutant protein. Difference in enzymatic activities between wild type and mutant protein was also monitored by HPLC analysis.

\section{Methods}

Site-directed Mutagenesis. To confirm the hPNMT binding site of the inhibitor, a K57A mutant was generated with the QuikChange ${ }^{\circledR}$ II XL site-directed mutagenesis kit (Stratagene, La Jolla, CA), using the pET-28a vector containing the PNMT gene (Novagen) as template DNA. The anti-sense primer, $5^{\prime}$ CAAGCAGCGCAGCGCCCACGGCCCGA-3', included the mutation. PCR was performed with a $50 \mu \mathrm{L}$ mixture containing $10 \mathrm{ng}$ template plasmid DNA, $125 \mathrm{ng}$ of each primer, $1 \mu \mathrm{L}$ of dNTP mix, $2.5 \mathrm{U}$ of cloned PfuUltra HF DNA polymerase in $0.5 \times$ PfuUltra HF DNA polymerase reaction buffer. The thermal cycler was programmed for amplification as follows: 18 cycles of denaturation at $95^{\circ} \mathrm{C}$ for $50 \mathrm{~s}$, annealing at $60^{\circ} \mathrm{C}$ for $50 \mathrm{~s}$, and extension at $68^{\circ} \mathrm{C}$ for $7 \mathrm{~min}$. Dpn I ( $\left.1 \mu \mathrm{L} ; 10 \mathrm{U}\right)$ was added to the resulting PCR product, followed by incubation at $37{ }^{\circ} \mathrm{C}$ for $1 \mathrm{~h}$. Dpn I-treated DNA $(2 \mu \mathrm{L})$ was used to transform XL10-GOLD ultracompetent cells. The mutation was confirmed by automatic sequencing at Macrogen Inc. (Seoul, Korea).

Recombinant Protein Expression and Purification. The gene coding for the mutant proteins hPNMT K57A was expressed in Escherichia coli BL21 (DE3) and purification of protein follows methods previously described. ${ }^{11}$ The correctly folded His-tagged protein was purified by HiTrap chelating column, and then reloaded onto second column, Superdex ${ }^{\mathrm{TM}} 75$ column (Amersham Bioscience). The collected protein fraction was exchanged with $20 \mathrm{mM}$ Tris-Cl, $500 \mathrm{mM} \mathrm{NaCl}$, pH 7.4 buffer. At each stage of the purification process, SDS-PAGE was applied to identify the hPNMT-containing fraction.

Fluorescence Analysis. A model RF-5301PC spectrofluorophotometer (Shimadzu, Kyoto, Japan) was used to estimate the binding constants of inhibitors with wild type and hPNMT K57A mutant. The hPNMT protein $(10 \mathrm{mM})$ was added to buffer (20 mM Tris-HCl, $500 \mathrm{mM} \mathrm{NaCl}$, pH 7.4, and $20 \mathrm{mM} \mathrm{SAM}$ ). Each inhibitor candidate (YPN010, YPN016 and YPN017) and known inhibitors (THIQ and 2-aminoindan) was titrated to a final protein: inhibitor ratio of 1:10. The sample was contained in a $2 \mathrm{~mL}$ thermostatted cuvette with excitation and emission path lengths of $10 \mathrm{~mm}$. Fluorescence quantum yields of hPNMT and ligand were determined by tryptophan emission. Samples were excited at $290 \mathrm{~nm}$, and emission spectra were recorded for light scattering effects from 290 to $450 \mathrm{~nm}^{12}$ We estimated binding constant $K_{b}$ using the following equation. ${ }^{13,14}$

$$
\log \left(\frac{F_{0}-F}{F}\right)=\log K_{b}+n \log [\text { inhibitor }]
$$

$F_{0}$ and $F$ represent fluorescence intensity from hPNMT at $342 \mathrm{~nm}$ in the absence and presence of inhibitor, respectively, while $\mathrm{n}$ is the number of inhibitor binding sites on the protein.

HPLC Enzymatic Inhibition Assay. The effects of K57A mutation on enzymatic activity of hPNMT was measured according to the HPLC monitoring described previously. ${ }^{11}$ Briefly, assay mixture contained SAM ( $80 \mathrm{mM})$, wild-type hPNMT and/ or K57A mutant. For determination of inhibitory activity, noradrenaline $(40 \mathrm{mM})$ was added and incubated for $15 \mathrm{~min}$ at $37^{\circ} \mathrm{C}$. Reactions were terminated with $20 \mathrm{~mL}$ perchloric acid. The supernatant fractions were centrifuged at $6000 \mathrm{rpm}$ for $5 \mathrm{~min}$, and $10 \mathrm{~mL}$ of sample solution was subsequently injected into the HPLC system maintained at $4{ }^{\circ} \mathrm{C}$ and the chromatogram was monitored for 20 minutes. As the control, $100 \%$ of noradrenaline is converted to adrenaline in the presence of wildtype hPNMT. The experiments were replicated at least three times and their results were compared to the wild-type protein.

STD-NMR Studies. Saturation transfer difference NMR (STD-NMR) experiments were performed at KBSI (Korea Basic Science Institute). Spectra were collected at $298 \mathrm{~K}$, both with and without saturation. ${ }^{15,16}$ In the presence of excess ligand, spectral differences primarily constituted resonances belonging to ligand protons bound to PNMT. The protein was saturated on-resonance at $-1.0 \mathrm{ppm}$ and off-resonance at $40 \mathrm{ppm}$, with a cascade of 40 selective Gaussian-shaped pulses of $50 \mathrm{~ms}$ duration and a $100 \mathrm{~ms}$ delay between each pulse in all STD-NMR experiments. The total duration of the saturation time was set to $2 \mathrm{~s}$. For STD-NMR experiments, $10 \mathrm{mM}$ mutant protein in $10 \mathrm{mM}$ Tris-Cl, $0.5 \mathrm{mM}$ DTT, pH 7.0, and $1.0 \mathrm{mM}$ of each inhibitor was added (protein: ligand ratio of $1: 100)$. In total, 1024 scans for each STD-NMR experiment were acquired, and a WATERGATE sequence used to suppress the water signal. A spin-lock filter (5 kHz strength and $10 \mathrm{~ms}$ duration) was applied to suppress the protein background.

\section{Results And Discussion}

SAM-dependent methyl transfer is one of the most extensive physiological reactions. Among the methyltransferases, PNMT participates in the terminal step of adrenaline synthesis from noradrenaline, and is used as a catecholamine biosynthetic marker and regulator of the central nervous system. A known inhibitor SK\&F 29661 and several inhibitors of PNMT accept hydrogen bonds directly with Lys57, Glu219, and Asp267 of hPNMT. ${ }^{3,17,18}$ Among them, a hydrogen bond between Lys 57 and inhibitor is critical for inhibitory activity against hPNMT. ${ }^{19,20}$

Fluorescence Studies and Binding Constants. The hPNMT protein contains six tryptophan residues and Trp221 is close to the binding site. In the presence of inhibitors, protein fluorescence was decreased. This quenching of fluorescence was used to estimate the binding constant. Binding constant, $K_{b}$, is defined as [complex]/[free protein] [free inhibitor]. ${ }^{13}$ The fluorescence intensity was altered with increasing inhibitor concentrations. These changes are attributed to the formation of a complex of protein and inhibitor.

To define the hPNMT binding site of YPN inhibitor candidates and validate the accuracy of our in silico screening model, we performed experiments with a K57A hPNMT mutant protein. The circular dichroism spectra of wild-type hPNMT, and K57A mutant were essentially identical, indicating that overall structure of hPNMT was not affected by this mutation (data not shown). Fluorescence titration curves for wild-type and K57A 
(A)

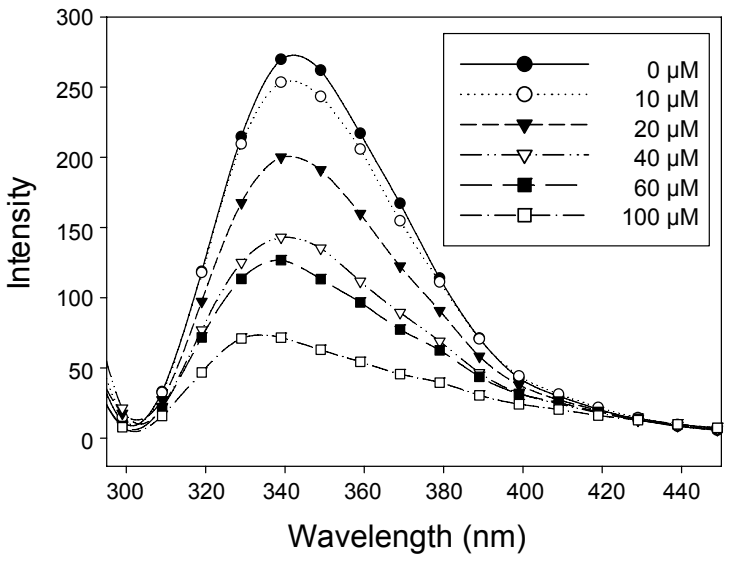

(B)

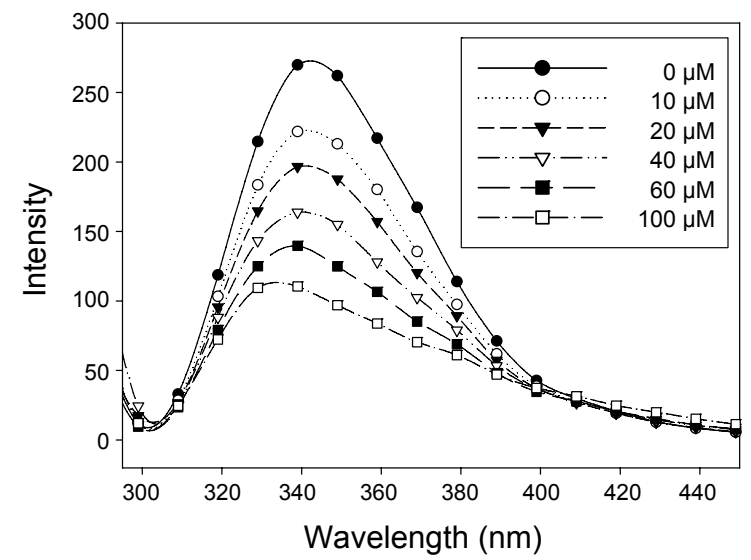

Figure 3. Fluorescence spectra of (A) wild-type hPNMT and (B) K57A mutant in the presence of different concentrations of YPN017. Samples were excited at $290 \mathrm{~nm}$ and emission spectra were recorded for light scattering effects from 295 to $450 \mathrm{~nm}$.

Table 1. Binding constants for wild type and mutant hPNMT for inhibitors

\begin{tabular}{cccccc}
\hline \multirow{2}{*}{$\begin{array}{c}\text { hPNMT } \\
\text { variant }\end{array}$} & \multicolumn{5}{c}{$K_{b}\left(\mathrm{M}^{-1}\right)$} \\
\cline { 2 - 6 } & YPN010 & YPN016 & YPN017 & THIQ & 2-aminoindan \\
\hline wild-type & $1.16 \times 10^{6}$ & $3.13 \times 10^{6}$ & $6.25 \times 10^{6}$ & $1.37 \times 10^{2}$ & 1.69 \\
K57A & $1.19 \times 10^{5}$ & $2.22 \times 10^{3}$ & $1.68 \times 10^{3}$ & $1.57 \times 10^{2}$ & 0.97 \\
\hline
\end{tabular}

mutant with YPN017 are depicted in Figure 3. As shown in Figure 3(A), significant decreases were evident in the quenching of wild-type hPNMT tryptophan fluorescence in the presence of YPN017. However, only a limited decrease in fluorescence quenching was obtained with their mutant form (Figure 3(B)), implying that the binding constant for mutant hPNMT with inhibitor was decreased dramatically. The binding constants for wild-type hPNMT and mutant are listed in Table 1. As shown in this table, the binding affinities of YPN010 $\left(K_{b}=\right.$ $\left.1.19 \times 10^{5} \mathrm{M}^{-1}\right)$ and YPN016 $\left(K_{b}=2.22 \times 10^{3} \mathrm{M}^{-1}\right)$ for the K57A mutant were reduced $\sim 10$ and $\sim 1400$ fold compared with wildtype, respectively. Strikingly, the greatest effect was demonstrated by the YPN017, resulting in reduction of binding affinities, $K_{b}$ from $6.25 \times 10^{6} \mathrm{M}^{-1}$ for wild type protein to $1.68 \times$ $10^{3} \mathrm{M}^{-1}$ for mutant protein. This data confirm that Lys 57 plays
(A)

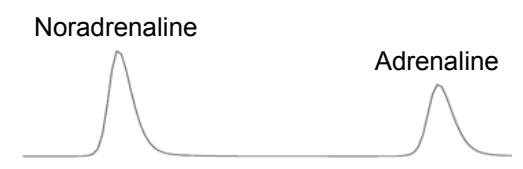

(B)

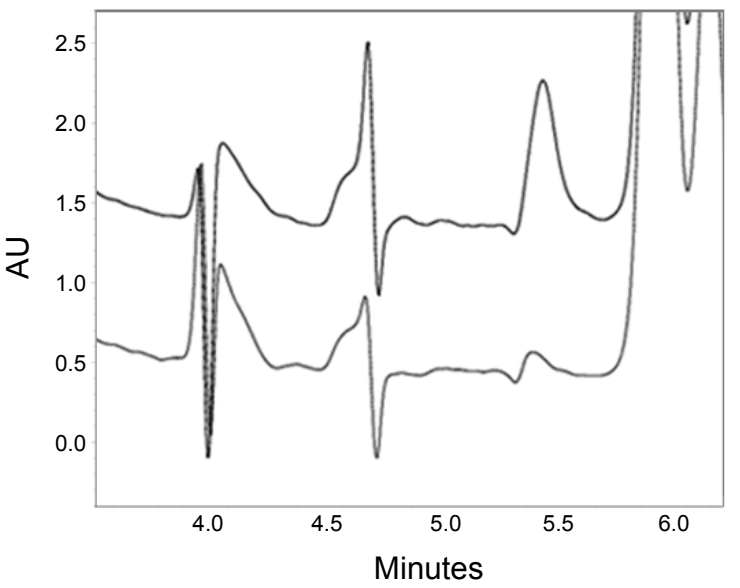

Figure 4. (A) HPLC separation of noradrenaline and adrenaline. (B) Stacked chromatograms of HPLC enzymatic activity assays for wild type (upper line) and K57A mutant hPNMT (lower line).

a key role in the binding of YPN inhibitors to hPNMT, consistent with our binding model. On the other hand, THIQ $\left(K_{b}=\right.$ $\left.1.57 \times 10^{2} \mathrm{M}^{-1}\right)$ and 2-aminoindan $\left(K_{b}=0.97 \mathrm{M}^{-1}\right)$ did not show any detectable decreased binding constants for K57A hPNMT, compared with that for wild-type protein as shown in Table 1. The crystal structure of hPNMT complexed with SK\&F 29661 which is an analog of THIQ, was reported earlier by Martin et $a$. $^{3}$ SK\&F 29661 has a sulfonamide substituent at the 7 position, which forms hydrogen bonds with Lys57 of hPNMT. ${ }^{3}$ Since THIQ does not have this sulfonamide group, it does not show significant change of binding constant by mutation of Lys57.

HPLC Enzymatic Activity Assay. To verify the importance of Lys57 residue in active site of hPNMT for interaction with inhibitors, we monitored the conversion of noradrenaline to adrenaline in the presence of hPNMT K57A mutant protein and in the presence of their wild-type counterpart, respectively, using a C18 column and a UV detector. As described previously, error values derived by reaction time and temperature are corrected by comparing the HPLC area with a control sample whereby only DMSO with no inhibitor was added. The HPLC enzymatic activity assay for YPN inhibitors with wildtype hPNMT reported previously, and YPN010, YPN016 and YPN017 exerted 100\% inhibition effects on the conversion from noradrenaline to adrenaline. ${ }^{11}$ As shown in Figure 4, compared to the wild type hPNMT, the K57A mutant showed a drastic reduction (95\%) in enzymatic activity of protein in the absence of any inhibitors indicating that Lys57 is key factor for catalytic reaction of hPNMT. Therefore, additional inhibitory effects of YPN inhibitors on enzymatic activity of K57A mutant were obviously not observed.

Biophysical Screening using NMR Spectroscopy. The STDNMR spectra of YPN016 in wild-type hPNMT and K57A 
(A)
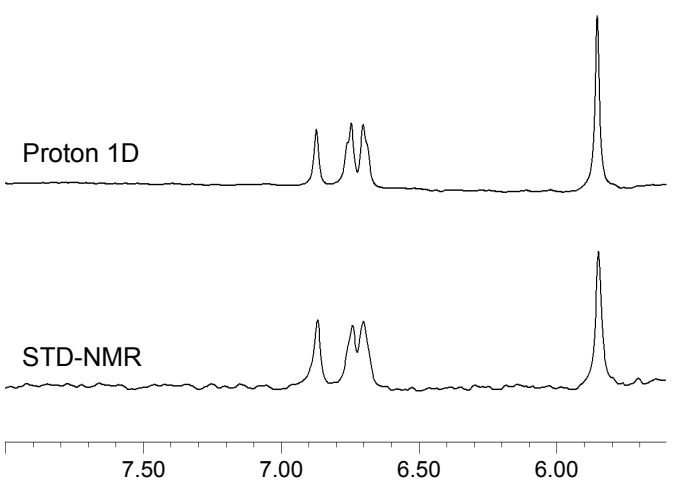

(B)

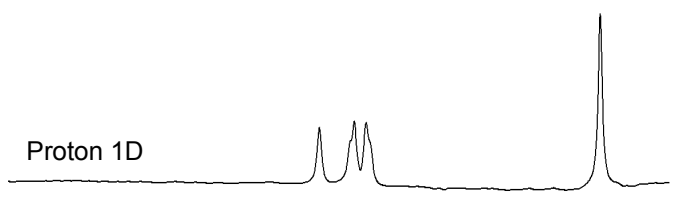

\section{STD-NMR}

7.50

7.00

6.50

6.00

Figure 5. Representative results of the STD-NMR binding assay. ${ }^{1} \mathrm{H}$ NMR spectra of YPN016 and its STD effects for (A) wild-type hPNMT and (B) K57A mutant hPNMT.

mutant are shown in Figure 5. In the presence of excess ligand, spectral differences primarily constituted resonances belonging to ligand protons bound to protein. STD-NMR spectra contained resonances specific for YPN016 bound to hPNMT (Figures 5(A), but there was no STD peak for the inhibitor bound to K57A mutant (Figure 5(B)). STD-NMR spectra of YPN016 proved that YPN016 binds well to wild-type hPNMT while it does not bind to the mutant protein and these results are in good agreement with fluorescence quenching results.

\section{Conclusion}

In our previous paper, we discovered potent inhibitors of hPNMT based on the in silico screening and enzymatic activity assay and we expected that hydrogen bond between Lys57 at the active site of hPNMT and inhibitors is an important feature for inhibition of protein. To confirm the importance of Lys57 of hPNMT for interaction with inhibitors, alanine mutagenesis was applied to a Lys57 of hPNMT and fluorescence quenching experiment and HPLC enzymatic activity assay was employed.

From HPLC enzymatic assay, K57A mutant did not convert the noradrenaline to adrenaline almost at all, resulting in complete suppression of enzymatic activity of hPNMT by muta- tion. It implies that Lys57 is key factor for catalytic activity of hPNMT. Also, the binding affinities of mutant protein to three YPN inhibitors were drastically reduced compared with wildtype. The STD-NMR experiments on YPN inhibitor added to the mutant protein suggested that Lys57 plays the key role on protein-inhibitor interaction. Our proposed inhibitor binding site including Lys 57 derived from in silico screening is in good agreement with the results derived from this mutational study.

Acknowledgments. This work was supported by the Korea Research Foundation (KRF-2006-311-C00394) and by the National Research Foundation of Korea (NRF) funded by the Ministry of Education, Science and Technology (2009-0076064).

\section{References}

1. Matsubara, K.; Aoyama, K.; Suno, M.; Awaya, T. Neurotoxicol. Teratol. 2002, 24, 593.

2. Berecek, K. H.; Brody, M. J. Am. J. Physiol. 1982, 242, H593.

3. Martin, J. L.; Begun, J.; McLeish, M. J.; Caine, J. M.; Grunewald, G. L. Structure 2001, 9, 977.

4. Axelrod, J. J. Biol. Chem. 1962, 237, 1657.

5. Wu, Q.; Gee, C. L.; Lin, F.; Tyndall, J. D.; Martin, J. L.; Grunewald, G. L.; McLeish, M. J. J. Med. Chem. 2005, 48, 7243.

6. Grunewald, G. L.; Seim, M. R.; Bhat, S. R.; Wilson, M. E.; Criscione, K. R. Bioorg. Med. Chem. 2008, 16, 542.

7. Grunewald, G. L.; Seim, M. R.; Regier, R. C.; Criscione, K. R. Bioorg. Med. Chem. 2008, 15, 1298.

8. Grunewald, G. L.; Seim, M. R.; Regier, R. C.; Martin, J. L.; Gee, C. L.; Drinkwater, N.; Criscione, K. R. J. Med. Chem. 2006, 49, 5424.

9. Grunewald, G. L.; Lu, J.; Criscione, K. R.; Okoro, C. O. Bioorg. Med. Chem. Lett. 2005, 15, 5319.

10. Grunewald, G. L.; Romero, F. A.; Criscione, K. R. J. Med. Chem. $\mathbf{2 0 0 5}, 48,1806$.

11. Kang, D. I.; Lee, J. Y.; Kim, W.; Jeong, K. W.; Shin, S.; Yang, J.; Park, E.; Chae, Y. K.; Kim, Y. Mol. Cells 2010, 29, 595.

12. Nissler, L.; Gebhardt, R.; Berger, S. Anal. Bioanal. Chem. 2004, $379,1045$.

13. Mishra, B.; Barik, A.; Priyadarsini, K. I.; Mohan, H. J. Chem. Sci. 2005, 117, 641 .

14. Azani, M-R.; Hassanpour, A.; Bordbar, A-K.; Mirkhani, V. Bull. Korean Chem. Soc. 2009, 30, 1973.

15. Mayer, M.; Meyer, B. J. Am. Chem. Soc. 2001, 123, 6108.

16. Macnaughtan, M. A.; Kamar, M.; Alvarez-Manilla, G.; Venot, A.; Glushka, J.; Pierce, J. M.; Prestegard, J. H. J. Mol. Biol. 2006, $366,1266$.

17. Gee, C. L.; Tyndall, J. D.; Grunewald, G. L.; Wu, Q.; McLeish, M. J.; Martin, J. L. Biochemistry 2005, 44, 16875.

18. Krovat, E. M.; Frühwirth, K. H.; Langer, T. J. Chem. Inf. Model. 2005, 45, 146 .

19. Gee, C. L.; Drinkwater, N.; Tyndall, J. D.; Grunewald, G. L.; Wu, Q.; McLeish, M. J.; Martin, J. L. J. Med. Chem. 2007, 50, 4845.

20. Lee, J. Y.; Jeong, K. W.; Kim, Y. Bull. Korean Chem. Soc. 2009, $30,1835$.

21. Grunewald, G. L.; Seim, M. R.; Regier, R. C.; Martin, J. L.; Gee, C. L.; Drinkwater, N.; Criscione, K. R. J. Med. Chem. 2006, 49, 5424.

22. Grunewald, G. L.; Borchardt, R. T.; Rafferty, M. F.; Krass, P. Mol. Pharmacol. 1981, 20, 377. 